\title{
Assisted dynamical Schwinger effect: pair production in a pulsed bifrequent field
}

\author{
Anatoly D. Panferov ${ }^{1}$, Stanislav A. Smolyansky ${ }^{1}$, Andreas Otto ${ }^{2,3}$, Burkhard Kämpfer ${ }^{2,3}$, David B. Blaschke ${ }^{4,5,6, a}$, \\ and Eukasz Juchnowski ${ }^{4}$ \\ 1 Department of Physics, Saratov State University, 410026 Saratov, Russia \\ 2 Institute of Radiation Physics, Helmholtz-Zentrum Dresden-Rossendorf, 01328 Dresden, Germany \\ 3 Institut für Theoretische Physik, Technische Universität Dresden, 01062 Dresden, Germany \\ 4 Institute for Theoretical Physics, University of Wroclaw, 50-204 Wroclaw, Poland \\ 5 Bogoliubov Laboratory for Theoretical Physics, Joint Institute for Nuclear Research, 141980 Dubna, Russia \\ ${ }^{6}$ National Research Nuclear University (MEPhI), 115409 Moscow, Russia
}

Received 15 September 2015 / Received in final form 2nd January 2016

Published online 15 March 2016

(c) The Author(s) 2016. This article is published with open access at Springerlink.com

\begin{abstract}
Electron-positron pair production by the superposition of two laser pulses with different frequencies and amplitudes is analyzed as a particular realization of the assisted dynamic Schwinger effect. It is demonstrated that, within a non-perturbative kinetic equation framework, an amplification effect is conceivable for certain parameters. When both pulses have wavelengths longer than the Compton wavelength, the residual net density of produced pairs is determined by the resultant field strength. The number of pairs starts to grow rapidly if the wavelength of the high-frequency laser component gets close to the Compton wavelength.
\end{abstract}

\section{Introduction}

The possibility of direct energy conversion processes from a strong electromagnetic field into $e^{-} e^{+}$pairs is one of the curious features of quantum electrodynamics (QED) [1-3]. However, the required critical electric field strength has the so-called Sauter-Schwinger value ${ }^{1} E_{c} \equiv m^{2} /|e|=$ $1.3 \times 10^{16} \mathrm{~V} / \mathrm{cm}$ (here, $m$ and $e$ are the mass and the charge of the electron, resp.) which makes it inaccessible to direct experimental observations at present. The hope for the observation of such processes was revived with the advent of ultra-intensity laser systems in the optical or X-ray regimes [4]. The rapidly evolving laser technologies [5] triggered repeatedly the theoretical search for suitable laser configurations which have the potential to realize pair production by Schwinger-type tunneling processes (for different variants, see $[6,7])$. A new avenue was provided by the dynamically assisted Schwinger effect $[8,9]$, meaning that the tunneling path is abbreviated by an assisting second field, thus enhancing the originally small tunneling probability. Given this scenario, a number of dedicated investigations aimed at further elaborating the prospects to find appropriate signals of the Schwinger effect.

Because of the important implications for related effects in other fields in physics (see Refs. [10,11] for an

\footnotetext{
${ }^{a}$ e-mail: blaschke@ift.uni.wroc.pl

1 We use $\hbar=c=k_{B}=1$ throughout this work.
}

overview including particle production in cosmology and astrophysics, Hawking-Unruh radiation as well as conceptional issues of vacuum definition), many investigations address either the principles of the strictly nonperturbative pair production [12] or employ special field models to elucidate the general features, often only by numerical evaluation.

The term "assisted Schwinger effect" stands for pair production from the vacuum under the influence of two fields - one assisting the other. Special field models are, for instance, particular pulses (such as the Sauter- or the Gauss-pulse) or oscillating fields with particular envelopes (such as Sauter- or Gauss-pulse with sub-cycle structures). Since in a spatially homogeneous electric field the threemomentum of a charged particle is a good quantum number which makes the mode expansion appropriate, one often restricts oneself to such homogeneous fields. The rationale for many models with a purely temporal dependence is that counter-propagating, suitably linearly polarized (laser) beams [13] in the homogeneity region of anti-nodes represent such spatially constant fields. The account for spatial gradients is quite challenging $[14,15]$ and requires much more efforts.

The enhancement of Schwinger type pair production by an assisting field has been considered already in references [7,16-24], e.g., for

(i) a constant field plus some pulse with or without subcycle structures [20]; 
(ii) a superposition of two pulses without sub-cycles [21];

(iii) a superposition of two pulses with oscillating subcycle structure [22-24].

In the latter case, the common envelope was taken with a long flat-top period with short ramping and de-ramping stages. Besides numerical examples, also the underlying enhancement mechanism has been clarified for that special field model: it is the shift of the relevant zero of the quasiparticle energy in the complex time domain toward the real axis (cf. [20,25] for other field configurations). Here we are going to extend the considerations in references $[23,24]$ and study, by numerical means, some systematics of the enhancement for a Gauss envelope. Besides the oscillation frequencies of both fields, the temporal width of the Gauss envelope enters as relevant new parameter related to time scales.

Our paper is organized as follows. In Section 2 we recall the formal framework of the quantum kinetic equations as basis of our non-perturbative analysis. In Section 3 we introduce the parametrization of the field model we consider. Numerical results are presented in Section 4. In Section 5 we give a critical discussion of the explored parameter range w.r.t. applications, and in Section 6 we present the summary of this work.

\section{Theoretical basis}

The non-perturbative consequence of the equations of motion of QED determines the vacuum effects in a given external, spatially homogeneous electric field with an arbitrary time dependence [26]. For instance, one can employ the quantum kinetic equation [27] describing the $e^{-} e^{+}$creation by an electric field $E(t)=-\partial_{t} A(t) \equiv-\dot{A}(t)$ with the four-vector potential in Hamilton gauge (we use natural units with $c=\hbar=1), A^{\mu}(t)=(0,0,0, A(t))$,

$$
\dot{f}(\mathbf{p}, t)=\frac{\lambda(\mathbf{p}, t)}{2} \int_{t_{0}}^{t} d t^{\prime} \lambda\left(\mathbf{p}, t^{\prime}\right) w\left(\mathbf{p}, t^{\prime}\right) \cos \theta\left(\mathbf{p}, t, t^{\prime}\right),
$$

where $w(\mathbf{p}, t)=1-2 f(\mathbf{p}, t)$ is the depletion function containing the dimensionless phase space distribution function per spin projection degree of freedom $f(\mathbf{p}, t)=$ $d N(\mathbf{p}, t) / d^{3} p d^{3} x$, and

$$
\lambda(\mathbf{p}, t)=\frac{e E(t) \varepsilon_{\perp}\left(p_{\perp}\right)}{\varepsilon^{2}(\mathbf{p}, t)},
$$

is the amplitude of the vacuum transition, while

$$
\theta\left(\mathbf{p}, t, t^{\prime}\right)=2 \int_{t^{\prime}}^{t} d \tau \varepsilon(\mathbf{p}, \tau)
$$

stands for the dynamical phase, describing the vacuum oscillations modulated by the external field. The quasiparticle energy $\varepsilon$, the transverse energy $\varepsilon_{\perp}$ and the longitudinal quasiparticle momentum $P$ are defined as:

$$
\begin{aligned}
\varepsilon(\mathbf{p}, t) & =\sqrt{\varepsilon^{2}\left(p_{\perp}\right)+P^{2}\left(p_{\|}, t\right)}, \\
\varepsilon_{\perp}\left(p_{\perp}\right) & =\sqrt{m^{2}+p_{\perp}^{2}} \\
P\left(p_{\|}, t\right) & =p_{\|}-e A(t)
\end{aligned}
$$

where $p_{\perp}=\left|\mathbf{p}_{\perp}\right|$ is the modulus of the momentum component perpendicular to the electric field, and $p_{\|}$stands for the momentum component parallel to $E$.

The integro-differential equation (1) is useful for the low-density approximation by setting $f\left(\mathbf{p}, t^{\prime}\right) \rightarrow 0$. For the complete numerical evaluations of (1) an equivalent system of ordinary differential equations is comfortable (arguments are dropped for brevity)

$$
\begin{aligned}
\dot{f} & =\frac{1}{2} \lambda u, \\
\dot{u} & =-2 \varepsilon v+\lambda(1-2 f), \\
\dot{v} & =+2 \varepsilon u,
\end{aligned}
$$

with $u$ and $v$ as auxiliary functions being related via $u^{2}+v^{2}+w^{2}=u^{2}+v^{2}+(1-2 f)^{2}=1$. Since the modes with momenta $\mathbf{p}$ decouple we have suppressed these arguments here, as well as the time dependence of all quantities. Sometimes, the relation $\dot{f}=\lambda u / 2$ is useful for a field acting a finite time only, telling that, since $E(t) \rightarrow 0$ implies $\lambda \rightarrow 0, f \rightarrow$ const for $E(t) \rightarrow 0$. Initial conditions at the remote past, $t_{0} \rightarrow-\infty$, are $u\left(t_{0}\right)=v\left(t_{0}\right)=f\left(t_{0}\right)=0$.

As emphasized, e.g., in [10], a sensible quantity is $\lim _{t \rightarrow \infty} f(\mathbf{p}, t)$, since the adiabatic particle number per mode depends on the chosen basis. Accordingly, the residual pair number density is

$$
n=\lim _{t \rightarrow \infty} 2 \int \frac{d \mathbf{p}}{(2 \pi)^{3}} f(\mathbf{p}, t) .
$$

The factor two refers to the two spin degrees of freedom which are summed up since in a purely electric field the spin degrees of freedom are degenerate.

Other formulations of the basic equations are conceivable, e.g., by relating $f$ to the reflection coefficient at (above) an effective potential, where the problem's heart is a Riccati equation $[20,25]$. In such a way the equivalence with a quantum mechanical scattering problem is highlighted, where the potential is related to $\varepsilon(\mathbf{p}, t)$. This makes evident that the residual phase space distribution can, in general, obey an intricate momentum dependence.

Asymptotic methods for the solution of the kinetic equation (1) were developed in $[28,29]$. There, some difficulties of applying such methods for field parameters corresponding to the case of tunneling regime are also discussed.

\section{Field models}

Only for a few cases the equations of Section 2 allow for exact solutions. Most notable are the Schwinger field 
$E_{\text {Schw }}=$ const and the Sauter pulse $E_{\text {Saut }} \propto 1 / \cosh ^{2}(t / \tau)$ with a time scale $\tau$. For a systematic approach to relate features of the residual momentum distribution and the temporal field shape, see [25]. Therefore, in most cases of interest, one has to resort to numerical solutions. Here one faces the problem that, for pulses with or without subcycle structures, a number of parameters determine the solution which can sensitively (often non-linearly) depend on the location in parameter space. Therefore, suitable approximations and estimates are very important. For instance, in a WKB type analysis the locations of zeroes of $\varepsilon$ in the complex $t$ plane are identified as important quantities determining the dominating exponential factor for the pair production. This also explains that pulses which look similar on the real $t$ axis can have strikingly different implications since the analytic properties can be rather distinctive. On a qualitative level, the enhanced pair production in the assisted dynamical Schwinger effect can be traced back to moving the relevant zeroes towards the real axis (cf. [20]), as mentioned above.

A subject of intense previous studies [30,31] was the Gauss pulse with sub-cycle structure or, equivalently, a periodic field with Gaussian envelope

$$
\begin{aligned}
E(t)= & E_{0} \cos (\omega t+\varphi) \exp \left(-\frac{t^{2}}{2 \tau^{2}}\right) \\
A(t)= & -\sqrt{\frac{\pi}{8}} E_{0} \tau \exp \left(-\frac{1}{2} \sigma^{2}+i \varphi\right) \\
& \times \operatorname{erf}\left(\frac{t}{\sqrt{2} \tau}-i \frac{\sigma}{\sqrt{2}}\right)+\text { c.c. }
\end{aligned}
$$

where $E_{0}$ is the amplitude, $\omega$ denotes the oscillation frequency and $\varphi$ is the carrier envelope phase, which determines the symmetry properties w.r.t. time reversal. Hereafter, we put $\varphi=0$. The parameter $\sigma=\omega \tau$ characterizes the number of oscillations within the pulse. For $\sigma>4$, the known examples [31] exhibit $f(t \rightarrow \infty)$ at $p_{\perp}=0$ as a strongly oscillating (in tune with $\tau$ ) function of $p_{\|}$around a bell-shaped mean, the latter one accessible via a WKB approximation. The occurrence of two time scales, $1 / \omega$ and $\tau$, allows to define two Keldysh parameters, $\gamma_{\omega}=(\omega / m)\left(E_{c} / E_{0}\right)$ and $\gamma_{\tau}=$ $1 /(m \tau)\left(E_{c} / E_{0}\right)$. Usually, $\gamma_{\omega} \ll 1$ is attributed [32] to the tunneling regime and can be termed dynamical Schwinger effect.

Considering (11) and (12) as the strong pulse in the spirit of the assisted dynamical Schwinger effect, one adds a second weak assisting pulse with the same envelope form but different parameters yielding an eight dimensional parameter space for the two-dimensional $p_{\perp}-p_{\|}$distribution. Here, the optimization theory $[19,22]$ is certainly very useful to search for parameters suitable for maximum amplification. Upon restricting to a narrow patch in the parameter space one can constrain the ansatz for the superposition of a strong and a weak pulse, each with sub-cycles, to

$$
\begin{aligned}
E(t)= & E_{0}\left\{\cos (\omega t)+k_{E} \cos \left(k_{\omega} \omega t\right)\right\} e^{-t^{2} /\left(2 \tau^{2}\right)}, \\
A(t)= & -\sqrt{\frac{\pi}{2}} E_{0} \tau \times\left\{\exp \left(-\frac{\tau^{2} \omega^{2}}{2}\right)\right. \\
& \times \operatorname{Re}\left[\operatorname{erf}\left(\frac{t}{\sqrt{2} \tau}+i \frac{\tau \omega}{\sqrt{2}}\right)\right] \\
& +k_{E} \exp \left(-\frac{\tau^{2}\left(k_{\omega} \omega\right)^{2}}{2}\right) \\
& \left.\times \operatorname{Re}\left[\operatorname{erf}\left(\frac{t}{\sqrt{2} \tau}+i \frac{\tau k_{\omega} \omega}{\sqrt{2}}\right)\right]\right\} .
\end{aligned}
$$

In these expressions, $k_{E} \leq 1$ is the field strength fraction of the amplitude of the weak pulse, and $k_{\omega} \geq 1$ is the frequency ratio. The envelopes of both pulses are synchronized and the carrier envelope phases are dropped, leading to a $t \rightarrow-t$ symmetric field $E(t)$. Thus, we are going to quantify the assisted dynamical Schwinger effect for moderate values of $k_{E}, k_{\omega}$ and $\tau$ in the mildly subcritical regime with $E_{0}<E_{c}$ and $\omega \leq m$. Having more extreme conditions in mind, e.g. $k_{\omega} \ggg 1$, another field model could be more suitable, such as

$$
E(t) \approx E_{0}\left(1+k_{E} \cos k_{\omega} \omega t\right) \times \text { envelope }
$$

and the related function $A(t)$. Besides the Gauss envelope, other pulse shapes and/or nonzero carrier envelope phases may be considered in separate work. Here we will just consider the example of the super-Gauss bifrequent field model

$$
E_{n}(t)=E_{0}\left\{\cos (\omega t)+k_{E} \cos \left(k_{\omega} \omega t\right)\right\} e^{-\frac{1}{2}(t / \tau)^{\nu}} .
$$

The Gauss envelope (13) is contained in (16) for the value $\nu=2$.

\section{Numerical results}

Figure 1 shows an example for the electric field (upper row) and the potential (lower row) of the strong, lowfrequency pulse (left column, field " 1 " characterized by $E_{0}, \omega, \tau$ in (11) and (12)), the weak, high-frequency pulse (middle column, field " 2 " characterized by $k_{E} E_{0}, k_{\omega} \omega$, $\tau$ to be used in (11) and (12) instead of $\left.E_{0}, \omega, \tau\right)$ and the superposition of both (right column, field " $1+2$ " according to (13) and (14)). We emphasize the much more pronounced "roughening" of the electric field " $1+2$ " by "2", while the impact on the potential looks very modest (note the different scales of left and middle panels in the bottom row). In Figure 3 we show the field (upper panel) and the potential (lower row) of the super-Gauss model in the case $\nu=8$ which gives the high frequency field "2" a shape with a flat top (see, e.g., Ref. [23]) and the wings of the combined field " $1+2$ " show a stronger modulation.

In Figure 2 (Fig. 4) we show the residual phase space distribution at $p_{\perp}=0$ (upper row) and $p_{\|}=0$ (lower row) 

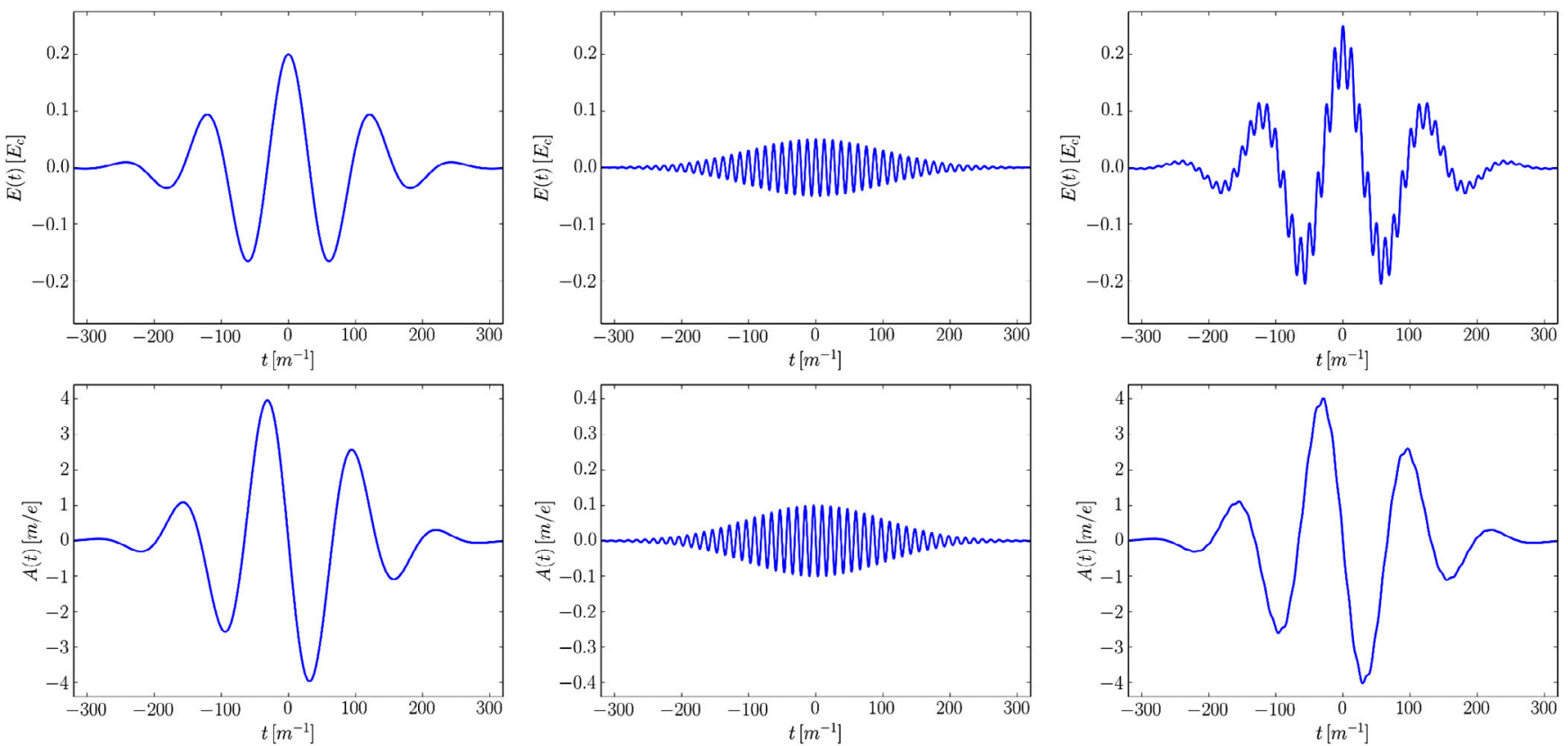

Fig. 1. The time dependence of the electric field (13) (upper row) and the potential (14) (lower row) for $E_{0}=0.2 E_{c}, \omega=0.05 m$, and $\tau=5 / \omega$. Left column: the strong, low-frequency component " 1 " of the pulse, i.e. only the first term in curly brackets in equations (13) and (14) corresponding to $k_{E}=0$; middle column: the weak, high-frequency component " 2 " with $k_{E}=0.25$ and $k_{\omega}=10$, i.e. only the second term in curly brackets in equations (13) and (14); right column: the superposition " $1+2 "$, i.e. the complete expressions in equations (13) and (14).
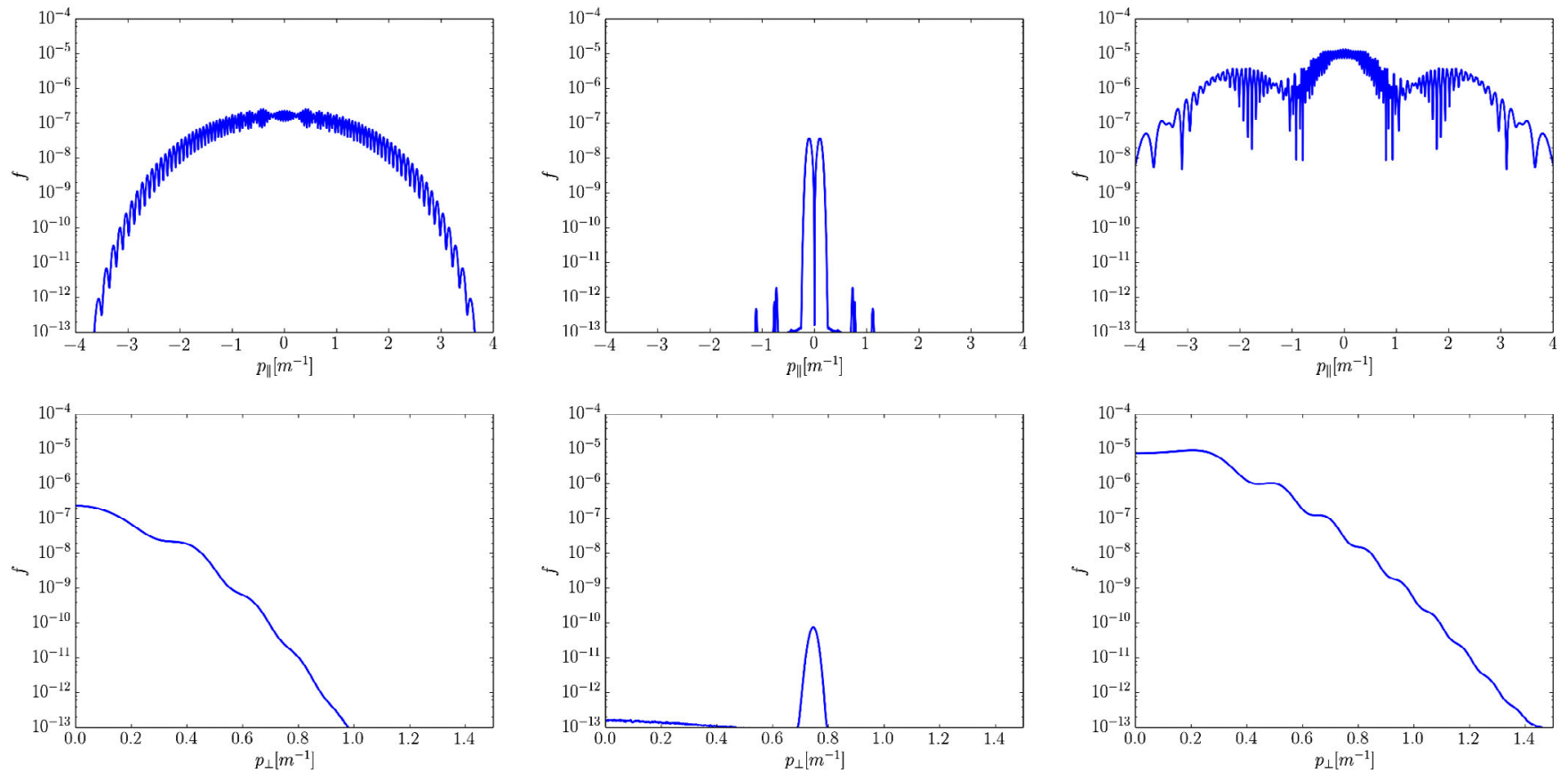

Fig. 2. Residual phase space distributions at $p_{\perp}=0$ (upper row) and $p_{\|}=0$ (lower row) for the fields displayed in Figure 1 . Left column: the strong, low-frequency component "1" of the pulse, i.e. only the first term in curly brackets in equations (13) and (14) corresponding to $k_{E}=0$; middle column: the weak, high-frequency component " 2 " with $k_{E}=0.25$ and $k_{\omega}=10$, i.e. only the second term in curly brackets in equations (13) and (14); right column: the superposition " $1+2$ ", i.e. the complete expressions in equations (13) and (14). 

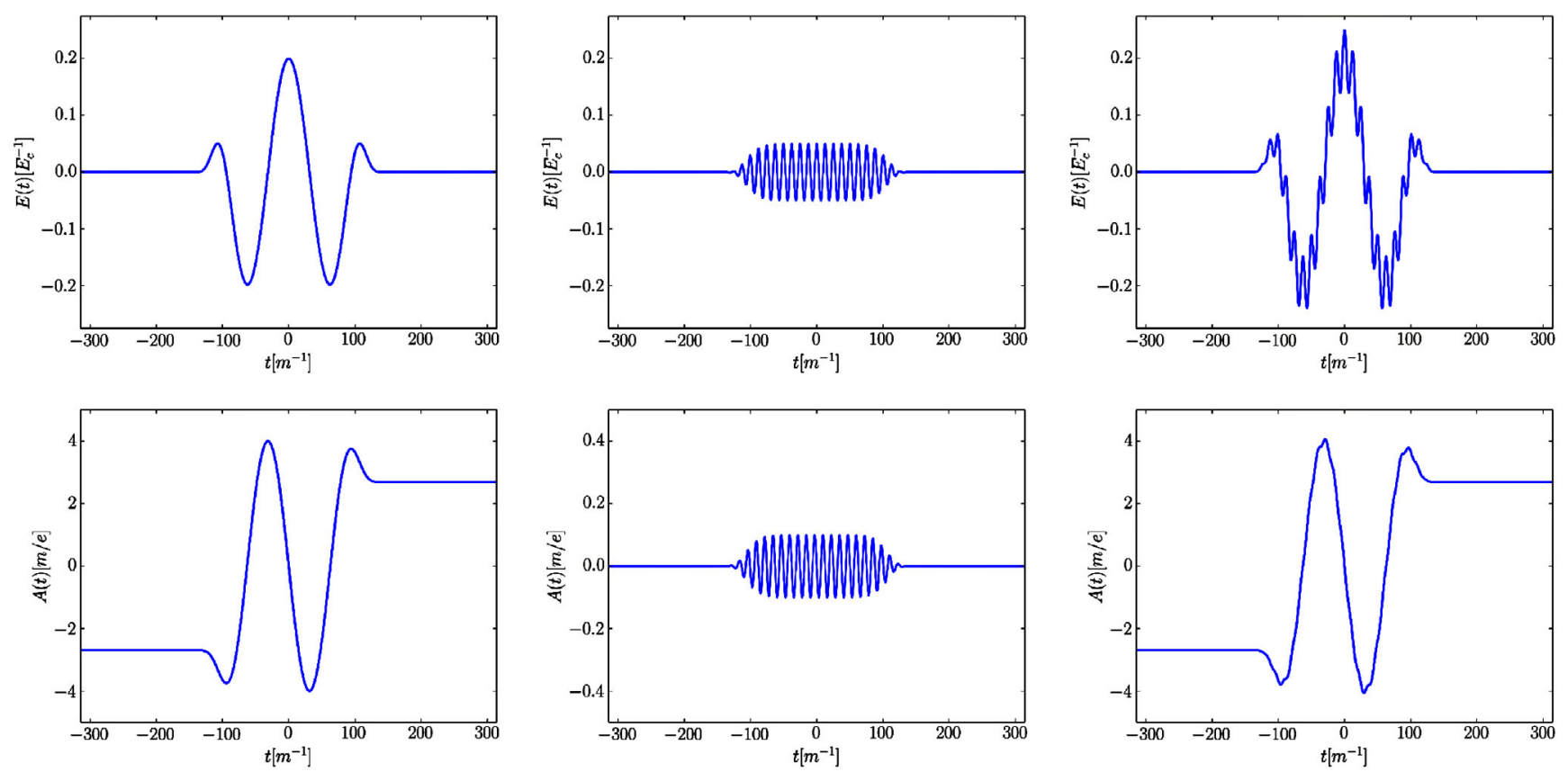

Fig. 3. The time dependence of the super-Gauss electric field (16) (upper row) and the potential (lower row) for $\nu=8$, $E_{0}=0.2 E_{c}, \omega=0.05 \mathrm{~m}$, and $\tau=5 / \omega$. Left column: the strong, low-frequency component " 1 " of the pulse $\left(k_{E}=0\right)$; middle column: the weak, high-frequency component " 2 " with $k_{E}=0.25$ and $k_{\omega}=10$; right column: the superposition " $1+2$ ".

for the fields displayed in Figure 1 (Fig. 3). It is obvious that here a nonlinear parametric enhancement effect takes place. The maximum values of the distribution function for the bifrequent pulse " $1+2$ " are almost two orders larger than the corresponding values for the low-frequency pulse " 1 " and almost three orders of magnitude for the highfrequency pulse " 2 ". In addition, the phase space occupancy for " $1+2$ " is apparently strikingly larger. Contrary to $[23,24]$, one can hardly recognize a "lifting" of the $p_{\|}$ distribution for field " 1 " by " 2 ": the patterns are fairly different. In the present case, there is no "flat-top" in the time dependence of the field envelope, but the crucial aspect for the observed enhancement is the dominance of pair production in the multiphoton regime for the weakfield, occurring for sufficiently large frequencies $k_{\omega} \omega$.

This situation is not qualitatively changed for the super-Gauss field with $\nu=8$ (see Fig. 3), where due to the flattening of the envelope shape resonant-like structures appear in the distribution function for the individual pulses while the combined bifrequent pulse results in a pair distribution function (shown in the lower right panel of Fig. 4) very similar to that for the Gaussian pulse shown in the lower right panel of Figure 2.

Due to the rather structureless behavior of the distribution function for the bifrequent fields of Gauss or superGauss type, the density (10) is easily accessible. Instead of $n$ we show in the following the dimensionless combination $N_{e^{-} e^{+}}=n / \omega^{3}$ which characterizes the number of pairs generated in a volume determined by the transverse size of the minimum focal spot attainable at the diffraction limit of field " 1 ".
Figure 5 shows the increase of the number of pairs created with increasing field strength $k_{E} E$ of the highfrequency pulse from small to large values of $k_{E}$. The left panel shows also a strong dependence of the effect on the frequency $k_{\omega} \omega$ of the second component of the field: at $k_{\omega}=10$ the amplification effect becomes noticeable only for $k_{E}>0.01$. For $k_{\omega}=40$, an enhancement effect is seen already for $k_{E}>0.0001$. Such a behavior has been noted already in reference [24] for another special field model and in reference [20] more generally: keeping fixed all other parameters, a certain value of the field strength " 2 " is required to cause a noticeable amplification by the assisting field. The right panel of Figure 5 shows that the effect is universal for different frequencies $\omega$ of the strong field " 1 ". The effect depends weakly on $\omega$ at fixed highfrequency $k_{\omega} \omega$. In the inset of that panel, we show the ratio $r=N_{e^{-} e^{+}}\left(k_{E}\right) / N_{e^{-} e^{+}}(0)=n_{1+2} / n_{1}$ as a function of $k_{E}$ to quantify the amplification effect. In particular, at $k_{e} \rightarrow 1$, the enhancement due to the assisting field becomes enormously large.

The dependence of the amplification effect on the frequency $k_{\omega} \omega$ of the weak, high-frequency field component is presented in Figure 6. In the left panel, the dependence of the number of created pairs is presented for three values of the strong-field frequency $\omega$. At the same time, the frequency range of the second field component runs in each case over a range from values of the frequency $\omega$, i.e. $k_{\omega}=1$, up to $k_{\omega} \omega=2 \mathrm{~m}$. The limiting case of equality of the first and the second frequency components is equivalent to an increase of the field amplitude of the first component by the coefficient $1+k_{E}$ and corresponds to the field 

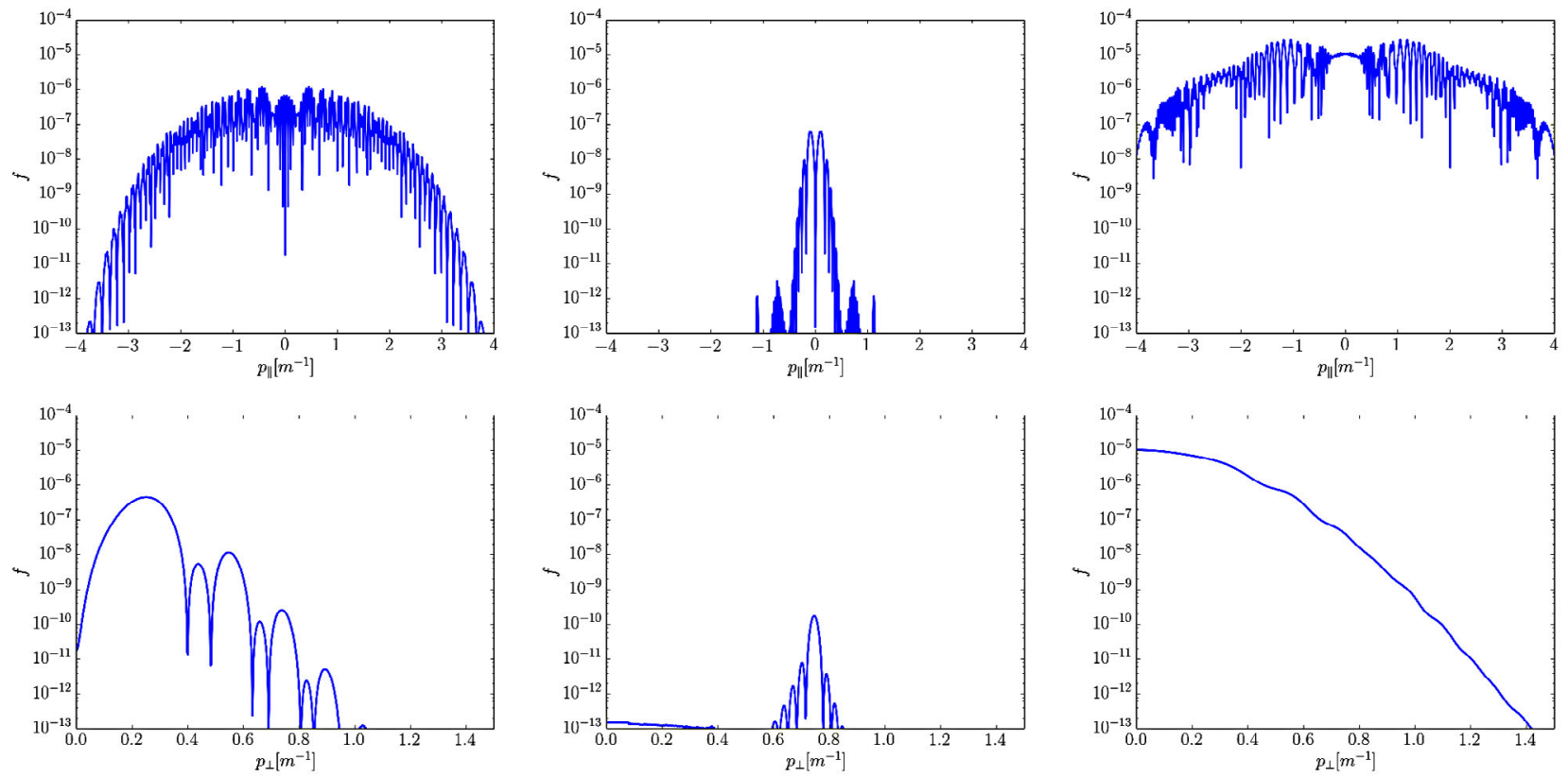

Fig. 4. Residual phase space distributions at $p_{\perp}=0$ (upper row) and $p_{\|}=0$ (lower row) for the fields displayed in Figure 3 . Left column: the strong, low-frequency component " 1 " of the pulse $\left(k_{E}=0\right)$; middle column: the weak, high-frequency component "2" with $k_{E}=0.25$ and $k_{\omega}=10$; right column: the superposition " $1+2$ ".
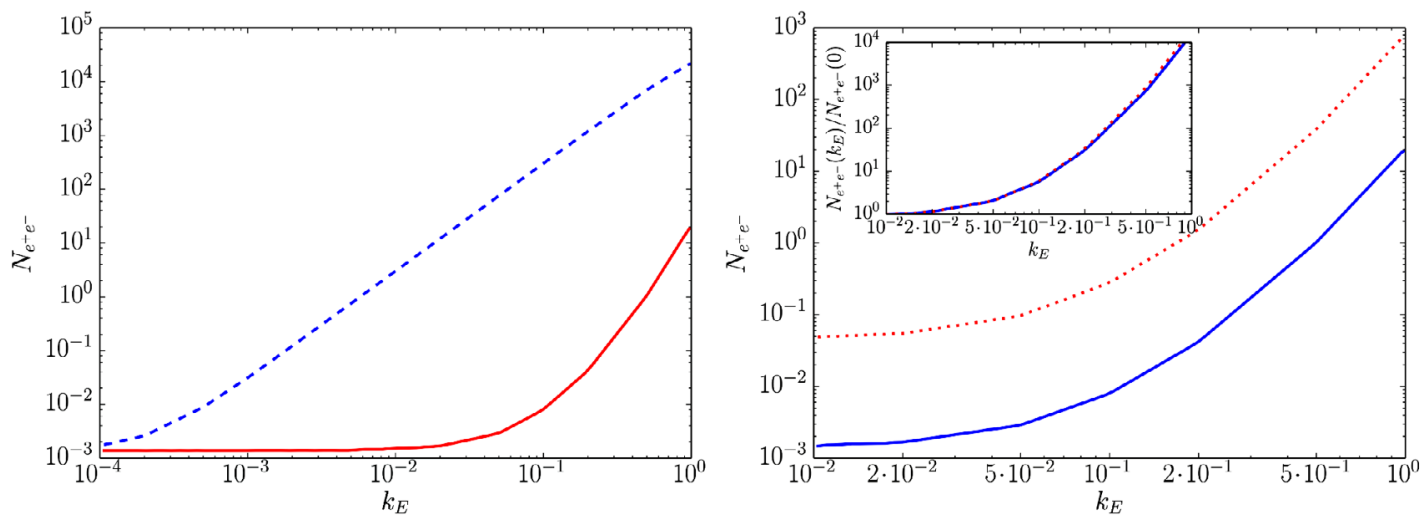

Fig. 5. The number of pairs produced in the pulse of equations (13) and (14) with $E_{0}=0.2 E_{c}$ and $\sigma=5$. Left: $\omega=0.05 \mathrm{~m}$ and $k_{\omega}=10$ (solid curve) and $k_{\omega}=40$ (dashed curve). Right: $\omega=0.05 \mathrm{~m}$ (solid curve) and $\omega=0.02 \mathrm{~m}$ (dotted curve); $k_{\omega} \omega=0.5 \mathrm{~m}$ for both curves. The inset shows the ratio $r=N_{e^{-} e^{+}}\left(k_{E}\right) / N_{e^{-} e^{+}}(0)=n_{1+2} / n_{1}$.
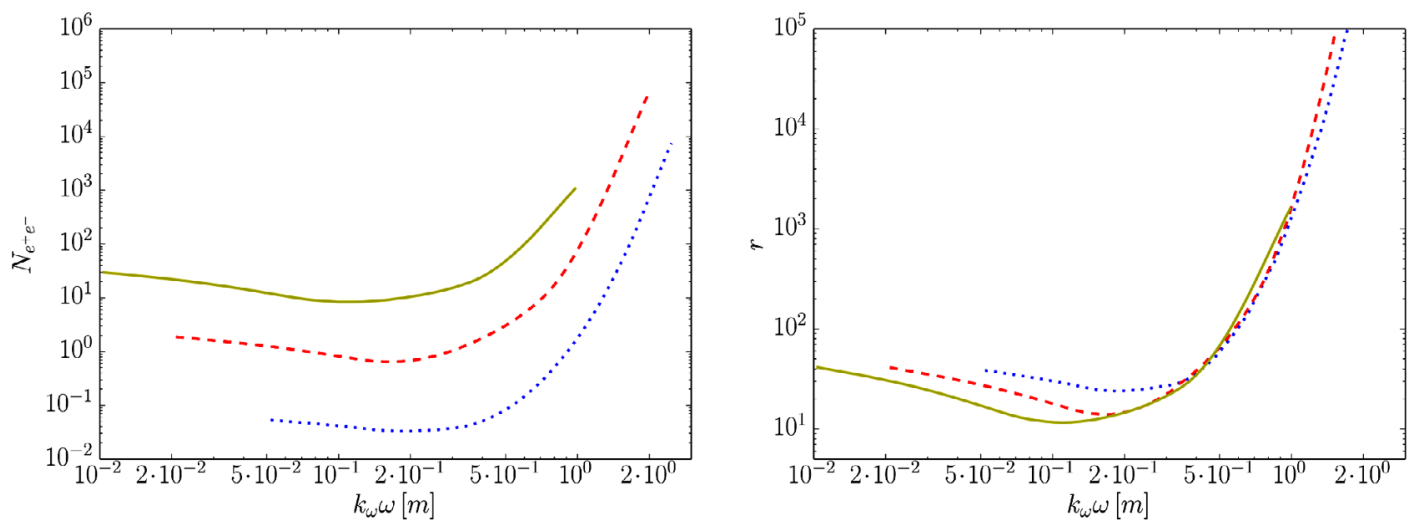

Fig. 6. Effectiveness of increasing the number of pairs produced for pulse type given by equations $(13)$ and (14) with $E_{0}=0.2 E_{c}$, $k_{E}=0.25$ and $\sigma=5$. The number of pairs $N_{e^{-} e^{+}}$(left) and the ratio $r$ (right) as a function of the weak-field frequency $k_{\omega} \omega$ for $\omega=0.01 \mathrm{~m}$ (solid curve), $0.02 \mathrm{~m}$ (dashed curve) and $0.05 \mathrm{~m}$ (dotted curve). 

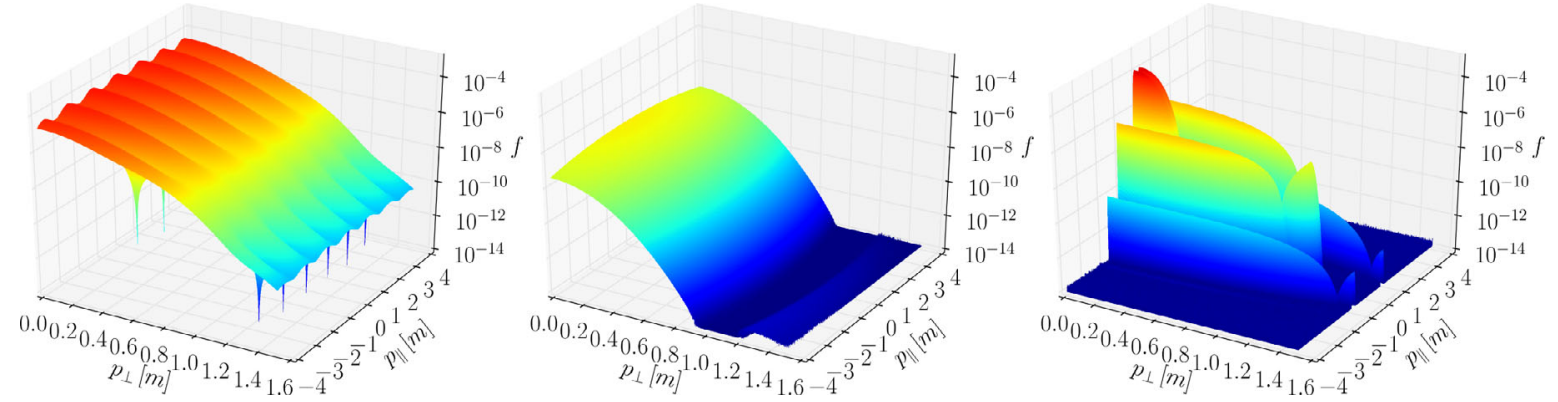

Fig. 7. The phase distribution for the pulse (15) with a Gaussian envelope with $E_{0}=0.2 E_{c}$ and $\sigma=50$. Left: $k_{E}=0$; middle: only for the second term in (15) with $k_{E}=0.25, k_{\omega} \omega=m$; right: $k_{E}=0.25, k_{\omega} \omega=m$.

defined by equations (11) and (12) with $E_{0} \rightarrow E_{0}\left(1+k_{E}\right)$. The right panel of Figure 6 shows the dependence of the ratio $r$ of the enhancement of pair creation by the second field component. For all three pulse frequencies $\omega$ the ratio shows these unique features:

(i) at $k_{\omega}=1$ the enhancement stems from a coherent superposition of the high and the low field which is quantitatively described by a simple rescaling of the field strength $E_{0} \rightarrow E_{0}\left(1+k_{E}\right)$ in the law of pair production by the single high field;

(ii) for high values of the low-field frequency $k_{\omega} \omega \gtrsim 0.5 \mathrm{~m}$ the results are almost identical and only weakly depending on the high-field frequency $\omega$, they are dominated by the multiphoton regime for the assisting weak field;

(iii) in between these two cases a dip in the ratio occurs due to the transition from coherent to incoherent superposition of the two fields.

It should be stressed that the pair production in the multi-photon regime becomes very efficient at high frequencies and depends less on the field strength than in the tunneling regime. To illustrate that point let us consider the pulse model (15) with a Gaussian envelope and $E_{0}=0.2 E_{c}$ and $\omega_{2}=m$. For $k_{E}=0$, i.e. only the first term in (15), the phase space distribution is smooth (see the left panel of Fig. 7), in contrast to the distribution shown in the left panel of Figure 2. For larger values of $\sigma$, the distribution approaches that of the Schwinger process, which is flat in $p_{\|}$direction and Gaussian shaped in $p_{\perp}$ direction. In the displayed momentum range, one pronounced multi-photon peak is visible when considering the second term in (15) alone, see the middle panel of Figure 7; it is accompanied by much lower side-ridges in $p_{\perp}$ direction (the cross section at $p_{\perp}=0$ looks similar to the middle panel of Figure 2, of course). Its peak value is much higher than the maximum seen in the left panel, even the field strength is less. That is the efficiency of the multi-photon process. The complete pulse (15) gives rise to the phase distribution exhibited in the right panel. The enhancement relative to the left panel is obvious, but the net effect falls short in comparison to the middle panel, when comparing the maxima of $f$. In this example the action of field " 2 " looks more like a "lifting" of the distribution emerging from " 1 ", albeit without the ripples. While the ratio $r=n_{1+2} / n_{1}$ rises strongly for $k_{\omega} \omega \rightarrow m$ (as seen in the right panels of Figs. 5 and 6 for another pulse), the net efficiency $n_{1+2} /\left(n_{1}+n_{2}\right)$ acquires a maximum which can be much larger than unity, but drops ultimately to unity upon enlarging further $k_{\omega} \omega$ as emphasized in reference [21]. It is the distinct phase space distribution which becomes important to discriminate the impact of the field components.

\section{Discussion}

Our investigation was originally motivated by the availability of XFELs $\left(E_{\text {XFEL }} \sim 10^{-5} E_{c}, \omega_{\text {XFEL }} \sim 5-50 \mathrm{keV}\right.$, cf. fig. 1 in [33] and [24]) and PW laser systems $\left(E_{\mathrm{PW}} \sim\right.$ $10^{-3} E_{c}, \omega_{\mathrm{PW}} \sim 1-3 \mathrm{eV}$, cf. [34-36]). These installations, when being combined with each other (as envisaged in the HIBEF project [37] for instance, or available already at LCLS [38]), in principle, would be characterized by $k_{\omega}>10^{3}$ and $k_{E} \sim 10^{-2}$. Moreover, pulse lengths of sub-attosecond duration would correspond to $m \tau \sim 10^{2}$. Clearly, these values are fairly distinct from those we have considered above. Thus, our present considerations do not directly apply to situations which can be expected to be exploited for experimental investigations towards the assisted dynamical Schwinger effect. In so far, our work is an exploratory supplement to studies searching for promising designs with discovery potential w.r.t. genuinely nonperturbative mechanisms of particle production. Without strikingly new ideas on avenues to the experimental verification of the Schwinger effect in freely propagating fields (in contrast to the nuclear Coulomb field), the many details understood by now call for significantly higher fields and/or large photon frequencies. Nevertheless, the facets of the Schwinger effect remain challenging, in particular due to their relation to many other fields as quoted in the Introduction.

\section{Summary}

When two pulses with different frequencies and different field strengths (the latter ones being high enough, not less than about an order of magnitude below $E_{c}$ ) one can talk about two mechanisms for the increase of the pair production. If the frequencies of the two components are close 
(in the extreme case, we even can assume they are the same) and are small compared to the energy required for multi-photon pair creation, the nature of the increment of residual pairs is directly related to the highly nonlinear dependence of the effect on the field strength in the vicinity of $E_{c}$. Alternatively, when one of the frequencies is not high and the second one is approaching the threshold of pair production by single photons we can talk about changing the properties of the vacuum for the high-energy photons. In this case, we can expect to more effectively promote the process of pair production and consider this process as pair production by a short-wavelength component catalyzing the low-frequency component.

In the present study we demonstrate that the increase of the rate of $e^{-} e^{+}$production by combining a strong lowfrequency field and a weak high-frequency field is a universal phenomenon and manifests itself in a certain range of parameters of the high-frequency field. Our results have been obtained within a non-perturbative framework. The shape of the electric field pulse is realistic and reproduces to some extent the characteristics of field pulses in experimental setups. The presented approach allows on the one hand to optimize the parameters for practical implementations of the dynamical Schwinger effect. On the other hand, by choosing parameters of the field model that characterise the actual experiment it allows to accurately estimate the number of residual pairs and their characteristics.

The authors acknowledge fruitful discussions with R. Sauerbrey, T.E. Cowan and D. Seipt within the HIBEF project. A.D.P. received support from the University of Wroclaw under internal number 2467/M/IFT/14 for his visit at the Institute for Theoretical Physics. D.B. and L.J. have been supported by Narodowe Centrum Nauki under grant number UMO-2014/15/B/ST2/03752.

\section{References}

1. F. Sauter, Z. Phys. 69, 742 (1931)

2. W. Heisenberg, H. Euler, Z. Phys. 98, 714 (1936)

3. J.S. Schwinger, Phys. Rev. 82, 664 (1951)

4. D.B. Blaschke, A.V. Prozorkevich, G. Röpke, C.D. Roberts, S.M. Schmidt, D.S. Shkirmanov, S.A. Smolyansky, Eur. Phys. J. D 55, 341 (2009)

5. A. Di Piazza, C. Müller, K.Z. Hatsagortsyan, C.H. Keitel, Rev. Mod. Phys. 84, 1177 (2012)

6. Conference on Extremely High Intensity Laser Physics (ExHILP), www.exhilp-heidelberg.de

7. A. Di Piazza, E. Löstedt, A.I. Milstein, C.H. Keitel, Phys. Rev. Lett. 103, 170403 (2009)

8. R. Schützhold, H. Gies, G. Dunne, Phys. Rev. Lett. 101, 130404 (2008)

9. G.V. Dunne, H. Gies, R. Schützhold, Phys. Rev. D 80, 111301 (2009)

10. R. Dabrowski, G.V. Dunne, Phys. Rev. D 90, 025021 (2014)
11. R. Ruffini, G. Vereshchagin, S.-S. Xue, Phys. Rep. 487, 1 (2010)

12. D.B. Blaschke, B. Kämpfer, S.M. Schmidt, A.D. Panferov, A.V. Prozorkevich, S.A. Smolyansky, Phys. Rev. D 88, 045017 (2013)

13. A. Di Piazza, Phys. Rev. D 70, 053013 (2004)

14. H. Kleinert, R. Ruffini, S.S. Xue, Phys. Rev. D 78, 025011 (2008)

15. C. Schneider, R. Schützhold, e-print arXiv:1407.3584

16. I. Akal, S. Villalba-Chávez, C. Müller, Phys. Rev. D 90, 113004 (2014)

17. Z.L. Li, D. Lu, B.S. Xie, L.B. Fu, J. Liu, B.F. Shen, Phys. Rev. D 89, 093011 (2014)

18. A. Nuriman, B.S. Xie, Z.L. Li, D. Sayipjamal, Phys. Lett. B 717, 465 (2012)

19. C. Kohlfürst, M. Mitter, G. von Winckel, F. Hebenstreit, R. Alkofer, Phys. Rev. D 88, 045028 (2013)

20. M.F. Linder, C. Schneider, J. Sicking, N. Szpak, R. Schützhold, Phys. Rev. D 92, 085009 (2015)

21. M. Orthaber, F. Hebenstreit, R. Alkofer, Phys. Lett. B 698, 80 (2011)

22. F. Hebenstreit, F. Fillion-Gourdeau, Phys. Lett. B 739, 189 (2014)

23. A. Otto, D. Seipt, D. Blaschke, B. Kämpfer, S.A. Smolyansky, Phys. Lett. B 740, 335 (2015)

24. A. Otto, D. Seipt, D. Blaschke, S.A. Smolyansky, B. Kämpfer, Phys. Rev. D 91, 105018 (2015)

25. C.K. Dumlu, G.V. Dunne, Phys. Rev. D 83, 065028 (2011)

26. A.A. Grib, S.G. Mamaev, V.M. Mostepanenko, Vacuum Quantum Effects in Strong External Fields (Friedman Laboratory Publish., St. Petersburg, 1994)

27. S.M. Schmidt, D. Blaschke, G. Röpke, S.A. Smolyansky, A.V. Prozorkevich, V.D. Toneev, Int. J. Mod. Phys. E 7, 709 (1998)

28. S.A. Smolyansky, M. Bonitz, A.V. Prozorkevich, Contrib. Plasma Phys. 53, 788 (2013)

29. S.A. Smolyansky, A.V. Prozorkevich, V.V. Dmitriev, A.V. Tarakanov, Int. J. Mod. Phys. E 23, 1450068 (2014)

30. F. Hebenstreit, R. Alkofer, G.V. Dunne, H. Gies, Phys. Rev. Lett. 102, 150404 (2009)

31. F. Hebenstreit, Ph.D. Thesis, Karl-Franzens-Universität Graz, 2011, arXiv:1106.5965

32. E. Brezin, C. Itzykson, Phys. Rev. D 2, 1191 (1970)

33. A. Ringwald, Phys. Lett. B 510, 107 (2001)

34. European Extreme Light Infrastructure (ELI), www. eli-laser.eu

35. High Power laser for Energy Research project (HiPER), www.hiper-laser.org

36. G.V. Dunne, Eur. Phys. J. ST 223, 1055 (2014)

37. The HIBEF project, www.hzdr.de/hgfbeamline

38. Linac Coherent Light Source (LCLS), ww.lcls.slac. stanford.edu

Open Access This is an open access article distributed under the terms of the Creative Commons Attribution License (http://creativecommons.org/licenses/by/4.0), which permits unrestricted use, distribution, and reproduction in any medium, provided the original work is properly cited. 